\title{
Mathematical Concepts and Physical Objects ${ }^{1}$
}

\author{
Giuseppe Longo \\ Labo. d'Informatique, CNRS - ENS et CREA, Paris \\ http://www.di.ens.fr/users/longo
}

\begin{abstract}
Introduction (with F. Bailly)
With this text, we will first of all discuss a distinction, internal to mathematics, between "construction principles" and "proof principles" (see [Longo, 1999], [Longo, 2002]). In short, it will be a question of grasping the difference between the construction of mathematical concepts and structures and the role of proof, more or less formalised. The objective is also to analyse the methods of physics from a similar viewpoint and, from the analogies and differences that we shall bring to attention, to establish a parallel between the foundations of mathematics and the foundations of physics. The paper is introduced by a joint reflection with a physicist, F. Bailly, coauthor of the complete French version of this work, originally a dialogue in two parts ([Bailly, Longo, 2005]).
\end{abstract}

When proposing a mathematical structure, for example the integers or the real numbers, the Cartesian space or ... a Hilbert space, we use a plurality of concepts often stemming from different conceptual experiences: the construction of the integers evokes the generalised successor operation, but at the same time we make sure they are "well-ordered", in space or time, to obtain this wellordered "line of integer numbers" which we easily "see", within a mental space. And we construct the rationals, as ratios of integers modulo ratio equivalence, and then the real numbers, as convergent sequences (modulo equiconvergence), for example. The mathematician "sees" this Cantor-Dedekind-styled construction of the continuum, a remarkable mathematical reconstruction of the phenomenal continuum. It is nevertheless not unique: different continuums may be more effective for certain applications, albeit that their structures are locally and globally very different, non-isomorphic to this very familiar standard continuum (see [Bell, 1998]). And this construction is so important that the "objectivity" of real numbers is "all there", it depends solely upon the well order of integers. One could say as much about the most important set theoretic constructions, the culmulative hierarchies of sets, the sets constructed from the empty set (a key concept in mathematics) by the iterated exponent operations, and so on... . These conceptual constructions therefore obey well-explicitated "principles" (of construction, as a matter of fact): successor, ordering in space (well order of integers, iteration, limits...)

But how may one grasp the "properties" of these mathematical structures? How may one "prove them"? The great hypothesis of logicism (Frege) as well as of formalism (Hilbert's program) has been that the logico-formal proof principles could have completely described the properties of the most important mathematical structures. Induction, particularly, as a logical principle (Frege) or as a potentially mecanisable formal rule (Hilbert), should have permitted to demonstrate all the properties of integers (for Frege, the logic of induction coincided, simply, with the structure of the integers - it should have been "categorical", in modern terms). Now it happens that logico-formal deduction is not even "complete" (let's put aside Frege's implicit hypothesis of categoricity); particularly, many of the integers' "concrete" properties elude it. We will evoke the "concrete" results of incompleteness from the last decades: the existence of quite interesting properties,

\footnotetext{
${ }^{1}$ In Rediscovering phenomenology (L. Boi, P. Kerszberg, F. Patras ed.), Kluwer, 2005 (Revised and translated version of Longo's part in [Bailly, Longo, 2005]).
} 
demonstrably realized by the numerical structures, and wich formal proof is unable to grasp. But that also concerns the fundamental properties of sets, the continuum hypotyhesis, and of the axiom of choice, for example, demonstrably true within the framework of certain constructions (Gödel, 1938), or demonstrably false (Cohen, 1964), so unattainable by the sole means of formal axiomatics and deductions.

To summarise this, the distinction between "construction principles" and "proof principles" shows that theorems of incompleteness prohibit the reduction (theoretical and epistemic) of the formers to the latters (or also of semantics - proliferating and generative - to strictly formalising syntax).

Can we find, this time, and in what concerns the foundations of physics, some relevance to such a distinction? In what would it consist and would it play an epistemologically similar role? Indeed, if the contents and the methods of these two disciplines are eminently different, the fact that mathematics plays a constitutive role for physics should nevertheless allow to establish some conceptual and epistemological correspondences regarding their respective foundations. This is the question we shall attempt to examine here. To do so, we will try to describe a same level of "construction principles" for mathematics and physics, that of mathematical structures. This level is common to both disciplines, because the mathematical structuration of the real world is a constitutive element of all modern physical knowledge (in short, but we will return to this, the constitution of the "physical object" is mathematical).

However, the difference becomes very clear at the level of the proof principles. The latter are of a logico-formal nature in mathematics, whereas in physics they refer to observation or to experience; shortly, to measurement. This separation is of an epistemic nature and refers, from a historical viewpoint, to the role of logicism (and of formalism) in mathematics and of positivism in physics. We will therefore base ourselves upon the following table:

\begin{tabular}{|l|l|l|}
\hline Disciplines & Mathematics & \multicolumn{1}{|c|}{ Côté physique } \\
\hline Construction principles level & Mathematical structures and their relationships \\
\hline Epistemic Reduction & Logicism/Formalism & Positivism/empiricism \\
\hline Proof principles level & Formal/Logical Languages & Experience/observation \\
\hline
\end{tabular}

Let's comment this schema with more detail. The top level corresponds to the construction principles, which have their effectiveness and their translation in the elaboration of mathematical structures as well as in the various relationships they maintain (that these structures be relative to mathematics as such or to the mathematical models which retranscribe, organise, and give rise to physical principles - and by that, partly at least, the phenomena that these principles "legalise" by provoking and often guiding experiments and observation). This community of level between the two disciplines, in what concerns construction, does not only come from the constitutive character of mathematics for physics, which we just evoked and which would almost suffice to justify it, but it also allows to understand the intensity of the theoretical exchanges (and not only the instrumental ones) between these disciplines. Either physics obtains elements of generalisation, modelisation, and generativity from mathematical structures and their relationships, or else physics' own developments suggest and propose to mathematics the construction of novel concepts...of which physics already make use, without waiting that they be rigorously founded. Historical examples abound: be it the case of leibnizian infinitesimals which appeared to be so paradoxical at the moment they were introduced - and for a long while after that - and which were never theoretically validated elsewise than by non-standard analysis, be it Dirac's "function" which was rigorously dealt with only in the theory of distributions, be it the case of Feynmann's path integrals - which 
have not yet found a sufficiently general rigorous mathematical treatment, while revealing themselves to be completely operable - or be it the birth of non commutative geometry inspired by the properties of quantum physics.

The bottom level, corresponding to that of the proof principles, divides itself into two distinct parts according to whether it concerns mathematics or physics (in that their referents are obviously different). For mathematics, what works as such are the corresponding syntaxes and logico-formal languages which, since Frege, Russell, Hilbert, have been presented as the foundations of mathematics. In fact, the logicism and formalism which have thus developed themselves at the expense of any other approach never stopped to identify the construction principles level with the proof principles level by reducing the first to the second. The incompleteness theorems having shown that this program could not be fulfilled for reasons internal to formalisms, the paradoxical effect was to completely disjoin one level from the other in the foundations of mathematics, by leading syntax to oppose semantics or by refusing to satisfy oneself with proofs not totally formalised (in the sense of this formalism) as can exist in geometry for example. In fact, it appears, conversely, that, as all of the practice of mathematics demonstrates, it is the conceptual coupling and circulation between these two levels that make this articulation between rigour and the innovative imagination which characterises the conceptual generativity of mathematics and the stability of its invariants.

Now a similar table can be drawn for physics, where the emergence of invariants (and symmetries) also constitutes a methodological turning point, as well as the constitution of objects and of concepts (see, for example, [Bailly, 2000], [Bailly, Longo, 2005a]). But this time, at the level of the proof principles, we no longer find a formal language, but the empiricism of phenomena: experiences, observations, even simulations, validate the theoretical predictions of mathematical models and prove their relevance. As constructed as they may have been by anterior theories and interpretations, it is the physical facts which constitute the referents and the instruments of proof. And there again, a particular philosophical option, related to the stage of development of the discipline and to the requirement of rigour in relation to physical factuality, has played, for the latter, a similar role to that of logicism and especially to that of formalism for mathematics. It consists in the positivism and the radical empiricism which, believing to be able to limit themselves only to facts, attempted to reduce the level of construction, characterised, namely, by interpretative debates, to that of proof, identified to pure empiricity. The developments of contemporary physics, that of quantum physics particularly, of course, but also that of the theory of dynamical systems, have shown that this position was no longer tenable and that the same paradoxical effect has lead, doubtlessly by reaction, to the epistemological disjunction between the levels of construction and of proof (a transposed trace is its opposition between "realists" and "nominalist" in the epistemology of physics). While, there again, all the practice of physicists shows that it is in the coupling and the circulation between these levels that lies the fecundity of the discipline. And, since for us the analysis of the genesis of concepts is part of foundational analysis, it is this productivity itself that feeds off interactions and which takes root within cognitive processes, which must be analysed.

It is thus in this sense, summarised by the above schema, despite their very different contents and practices, that the foundations of mathematics and the foundations of physics can be considered as presenting some common structural traits. That is, this distinction between two conceptual instances are qualifiable in both cases as construction principles and as proof principles, and the necessity of their coupling - against their disjunction or conversely, their confusion - is important to also be able to account for the effective practice of researchers in each of these disciplines. Moreover that they share the same level of mathematical structures characterising the dynamics of construction principles and feeding off the development of each of them. 
If we now briefly address the case of this other discipline of natural sciences which is biology, it appears, in what concerns the structure of its own foundations, to distinguish itself from this schema, though we may consider that it shares with physics the same level of proof principles, that is, the constraint of reference to the empiricity of observation and of experience. However, we are lead, at the level of this proof principle, to operate a crucial distinction between what is a matter of in vivo (biological as such in that it is integrated and regulated by biological functions), and what is a matter of in vitro (and which practically confounds itself with the physico-chemical). But what manifestly changes the most depends, it seems, on two essential factors. On one hand, the level of what we may call (conceptual) "construction principles" in biology still does not seem well characterised and stabilised (despite models of evolution, autonomy or autopoiesis). On the other hand, it seems that another conceptual level adds itself, one specific to the epistemology of the living, and to which is confronted any reflection in biology and which we may qualify, to use Monod's terminology, as the level of the teleonomic principle. This principle in some way makes the understanding of the living depend not only upon that of its past and current relationships to its relevant environment, but also upon that of the anticipations relative to the future of what this environment will become under the effect of its own activity of living (an aspect of this third factor of temporality, shown in [Bailly, Longo, 2003]). And this temporality lays itself beside the usual physical temporality which regulates the physico-chemical action-reaction relation and the biological temporality specific to the organism which manifests itself as the existence and the activity of "biological clocks" which time its functions (also see [Bailly, Longo, 2003]). This conceptual situation then leads to consider, for biology, the characterisation of an extra, specific concept, in interaction with the first two, which one of the authors called "contingent finality"; meaning by that the regulations induced by the implications of these anticipations, and which themselves open the way to the accounting for "significations" (see [Longo, 2003]).

This paper contains no more remarks on systems of life: some work in this direction may be found in [Bailly, Longo, 2003 and 2005a].

\section{Genealogies of concepts.}

Let's more closely tackle now the idea of a parallel between the constitution of mathematical concepts and of physical objects. We will only be able to respond partially to this inquiry and shall rather reflect upon the meaning of the relativising constructions specific to mathematics and to physics, within an explicative and foundational framework inspired by the arisen questions. But the project is wider, because it is a question of grounding the two "constitutive histories" within our worldly living being, to grasp this biological and historical "cognitive subject", which we share and which guarantees us the objectivity of our forms of knowledge. It is not a question of unifying by force the epistemologies of differing disciplines, but to make them "exchange between themselves", to reveal the reciprocal dependencies, the several common roots. The analysis we propose here will thus base itself upon the following principles:

- The problem of the foundations of mathematics is (also) an epistemological problem,

- $\quad$ Any epistemology (of mathematics) must refer to a conceptual genesis, as a "process of construction of knowledge"

- The epistemology of mathematics is an integral part of the epistemology of the sciences (the exact sciences, at least)

- A constitutive element of our scientific knowledge is the relationship, established in the different sciences, to space and to time.

In short, a sensible epistemology of mathematics must try to explicitate a "philosophy of nature", term which is dear to the great minds of the XIXth Century. As it is, mathematics are one of the 
pillars of our forms of knowledge, they help to constitute the objects and the objectivity as such of knowledge (exact knowledge), because they are the locus where "thought stabilises itself"; by this device, their foundation "blends" itself to other forms knowledge and to their foundations.

Moreover, the conceptual stability of mathematics, their relative simplicity (they can be profound all the while basing themselves upon stable and elementary, sometimes quite simple, principles) can provide the connection which we are looking for with the elementary cognitive processes, those which reflect some of the world's regularities in our active presence within that same world, as living beings (and living in intersubjectivity and in history). For these same reasons, the theories of knowledge, from Plato to Descartes, to Kant, Husserl or Wittgenstein, have all addressed the question of the foundations of mathematics, this "purified" knowledge", both mysterious and simple, where notions of "truth" and of "proof" (reasoning) are posed with extreme clarity. The problem of the cognitive foundations of mathematics must therefore be analysed as an essential component of the analysis of human cognition. Within that framework, we will attempt to analyse in what sense "foundations" and "genesis" (cognitive and historic) are strictly related. The very notion of "cognitive foundations" explicitly juxtaposes foundations and genesis (see [Longo, 2005] for more in this direction).

In this study, the notions of time and space which we use do not refer to "natural entities", but rather to the play between sensible experience and conceptual frameworks which allow the natural sciences to manifest themselves. That was in fact the inquiry of the great geometers (Riemann, Helmholtz, Poincaré, Enriques, Weyl ...) who tried to pose the problem of the foundations of mathematics within the framework of a philosophy of nature. But the analysis which came to dominate afterwards stemmed from a very clear division between logical (or formal) foundations and epistemological problems, particularly under the form of this relationship to time and space which ground mathematics in this world.

Frege explicitly denounces the "delirious" situation in which the problem of space finds itself, because of the emergence of non-Euclidean geometries ([Frege, 1884]), and proposes a "royal way out", by laying the bases of a new discipline, mathematical logic. Mathematics themselves are the development of "absolute laws of thought", logical rules outside of this world and independent of any cognitive subject. For that, Frege introduces a very clear distinction between "foundations" and "genesis", he breaks any epistemological ambition, all the while attacking "psychologism" (as of Herbart/Riemann) and "empiricism" (as of Stuart-Mill). The former try to understand which "hypotheses" (which "a priori") allow to make physical space (and time) intelligible to the knowing subject, while the latter relates mathematics to a theory, alas too naïve, of perception. Faced with all these first attempts at a "cognitive analysis" of mathematics, Frege proposes a philosophy centred upon a very inflexible dogma, the logicist dogma, according to which mathematics have no psychologico-historical or empirical genesis. They are, according to him, a constituted knowledge, concepts without conceptors. This philosophy, this dogma, is at the origin of the fundamental split, which will accompany all of the XXth Century, between foundational analysis and epistemological problems, between mathematics and this very world they organise and make intelligible ${ }^{2}$.

Moreover, for Frege, geometry itself, as given by numerical ratios ([Frege, 1884]), bases itself on arithmetics; and the latter is but the expression of logical laws, because the concept of the number is a logical concept and induction, a key rule of arithmetics, is a logical rule. Finally, the continuum, this difficult stake of phenomenal time and space, is also very well mathematised, in Cantor-Dedekind style, from arithmetics.

\footnotetext{
${ }^{2}$ For us, however, the "almighty dogma of the severance of principle between epistemological elucidation and historical explicitation as well as psychological explicitation within the sciences of the mind, of the rift between epistemological origin and genetic origin; this dogma, inasmuch as we do not inadmissibly limit, as it is often the case, the concepts of "history", of "historical explicitation" and of "genesis", this dogma is turned heads over heels" [Husserl, 1933: p.201].
} 
So there are the problems of time and space and of their mathematisation, neglected to the benefit of their indirect foundation, via arithmetics, upon logic; pure concepts, with no relationship whatsoever to sensible experience nor to physical construction. Conversely, this relationship was at the centre of the inquiry of the inventors of non-Euclidean geometries: Gauss, Lobatchevsky or Riemann did not play the logical negation of Euclid's Vth axiom and of its formal developments, but they proposed a "new physics", a different organisation of the world (see [Lobachevskij, 1856], [Riemann, 1854].) It also happens that the numerical relationships may possibly "found" Euclidean geometry, but surely not other geometries, because Euclidean geometry is the only one which "preserves" these relationships (it is the only one whose group of transformations - of automorphisms - which defines it, contains the homotheties ${ }^{3}$.)

Now it is doubtless that mathematics have a logical as well as a formal foundation (a distinction will need to be made here), but they are in fact a "three-dimensional" construction. They constitute themselves within the interactions of the logical and totally essential "if ...then" (1rst dimension), of perfectly formal, even mechanic calculus (2nd dimension), but also in a third conceptual dimension, these constructions of (and in) time and space, which mingle it, even moreso than the two others, to the different forms of knowledge. And the epistemological problem then poses itself as an analysis of the constitution of the invariants of language and of proof, these invariants which we call "logic" and "formal systems", as well as the invariants of time, and of space, upon which we construct our geometries, these "human constructs ... in our spaces of humanity" as Husserl says in the "Origin of Geometry" (see below). The problem is thus posed from the analysis of this very peculiar form of knowledge which is mathematics, from their cognitive roots, be they pre-human, to their communicable display, with its thousands of mediating levels.

Axiomatic conventions and logico-formal proof are actually but the ultimate results of a constitution of meaning, common notations of concepts rooted in "our living practices", to put it as Wittgenstein would do, in our "acts of experience" (Weyl): logico-formal analysis is a necessary accompaniment to this latter part of the epistemological process, the analysis of proof, of certain proofs, but it is insufficient (it is essentially "incomplete", some theorems tell us.) The foundational analyses of mathematics must thus be extended from the study of deduction and of axiomatics to that of the constitution of concepts and of structures; impossible without a parallel analysis of the constitution of the physical object and of perception.

\section{Concerning the "transcendent" in physics and in mathematics}

There is no doubt that there exists a reality beyond ourselves, which enters into "friction" with our actions upon it and which, moreover, "canalises" them. Husserl uses a word from the idealist tradition to designate this reality: he recourses to the notion of transcendence. In a very common interpretation of this word, and quite independently from Husserl, the following deduction is usually made, first in physics, then in mathematics: the "properties" of the world (physical, numerical, mathematical...) are transcendent and, moreover, are not all known. They are therefore "already there", they pre-exist. The objects of the world around us have well established properties that are quite stable and invariable in connection to our senses: I look at this pencil, I touch it, even its odour confirms its "objectivity", independently of the specific sense I use to explore it..., it is

\footnotetext{
3 Hilbert, as a great mathematician, will manage quite well otherwise. Thanks to the Beltrami-Klein interpretation of non-Euclidean geometries within Euclidean geometry, he will give a correct immersion (interpretation) of his axioms for geometry within arithmetics via analysis [Hilbert, 1899]. But, for the latter, he will not look for a "logical meaning", unlike Frege. Indeed, once geometry (ies) is (are) interpreted within arithmetics, a finitary proof of its (thus, their) coherence (of non-contradiction) would suffice for its foundational analysis, entirely and exclusively centred around its problem of coherence; its a pity it doesn't work, because arithmetics does not have, itself, any arithmetic (finitary) proof of coherence. To the contrary, we manage with an infinite piling of infinites, or by proof founded upon geometrical judgements, [Longo, 2002].
} 
thus already there, it pre-exists my explorations, with all its properties. In a completely analogous manner, the properties of numbers, of mathematical structures do not depend on notation (for numbers: decimal, binary...) nor on other details of representation, of the mathematician subject exploring them... therefore they pre-exist.

Now it is the word "property" - in physics, in mathematics - that must first be agreed upon: a property is "talked about", it is first of all an expression in these languages through which we try to speak of the world, to organise it and to give it meaning, a meaning shared with others. But the world canalyses our efforts to obtain knowledge and displays some "resistance" (causes friction) to our propositions to organise it. "Properties", as we render them through intersubjectivity by words, are not in themselves isomorphic to "absolute facts" that are "already there", possibly well established or that would manifest themselves under well established forms of linguistic structures; by our active gaze, in our exchange with others, we propose a structure with hints of a reality which is there, as unorganized frictional matter. Thus, through language, pictures, gesture, we unify certain phenomena, we draw contours upon a phenomenal veil which is an interface between the world and us. The transcendent is a constituted, it is the result of a constitutive activity, of a process which precedes the individual or that the individual performs mostly with others. This process is best synthesised as the result of a transcendental (and not transcendent) activity, and such is the lesson we draw from Husserl.

It is no coincidence if the many "examples of objects" proposed by ontologising philosophies, in mathematics, in physics, refer to "medium size manufactured objects", all the while attempting to escape the problem of cognitive relativism. These thinkers of ontology, of essences, rarely refer to the "objects" of quantum physics, for example, in order to propose an ontology that is much more difficult to take on, of the electron, of the photon ... But even these medium size manufactured objects, of an apparently such simple ontology, if it is true that they are really there, are just as much constituted as the concept they are associated to. The pencil is constructed, in history, at the same time as the "concept" of the pencil. Both are related to drawing, to writing, as human activities. They are pre-existent, the object and the concept, for the individual subject, they are not so for humanity, in its history. There was no pencil, nor table, nor a pot such as the one laid on Kurt Gödel's table, before the beginning of our human acting and thinking. On the other hand, there was surely already a physical "reality" (for Galileo, less so for Tales), but its organisation and its interpretation as photon, electron ... solid, stable, in fact mathematical, was not yet there, nor was it's organisation into pots, pencils, and tables before the blossoming of our humanity. And this approach, we think, does not face the dangers of relativism, because the objectivity of the constructed, of the concept, of the object, lies in the constitutive process which is itself objective.

Cassirer, quoted by Parrini in a work whose goal is to overcome the fracture between absolutism and relativism, partially addresses this theme [Parrini, 1995; p. 118]: " if we determine the object not as absolute substance beyond all knowledge, but as object which takes form whithin the progression of knowledge itself", then, "this object, from the viewpoint of the psychological individual, can be said to be transcendent", despite that "from the viewpoint of logic and of its supreme principles", it must "be considered as immanent." Ideality, the concept as "conceived", "a cut-out" ("decoupage") performed upon the world in order to give it contours, to structure it, will thus detach itself from subjective representation, despite that it may have its origins within the community of subjects, in what they share: similar bodies and brains from the start, in the same world, and all that which they build in common, in their common history. It is thus not a question of writing a history of individuals, but to trace back the origin of an idea; no historising relativism, but a reference to history as an explicitation of our "being together in the world", locus of the active constitution of all our forms of knowledge.

In the case of the objects of physics, of microphysics in particular, this activity of the construction of objects by "conceptual carving" is rather clear: electrons, muons, fermions, quantum fields ... are not already there. They are concepts that are proposed in order to unify, to 
organise, to understand the signals the world sends us. These signals are not arbitrary and they are also the result of an active exploration. In order to obtain them it was necessary to develop rather complex measurement instruments, which are themselves the result of a theory. All the instruments for physical measurement, and moreso those of microphysics, are constructed after an enormous theoretical commitment: I want to measure this but not that, by using these materials but not other ones, I "look" here and not there. The "facts" which result from this, as Goodman would say, are thus "small-scale theories" themselves.

Let's consider for example the wave-particle duality in quantum physics. The photon, the electron, present themselves as "waves" or "particles" depending upon the "experimental context": specific instruments are put into place, in fact the experiment is prepared from the viewpoint of a certain theory... . The object that will result from this will depend as much upon the theoreticoexperimental framework as it will upon friction - "the canalisation of thought" that nature imposes upon and through these tools. A certain viewpoint will show us the particle, another will show us the wave. More precisely, we will obtain macroscopical properties on a screen, on any detecting device, and by a process just as important, we will interpret them as "symptoms" of the "existence" of a particle or of a wave. There is no duality as such for the physical object, but a context of reconstruction of the world where we are as present as the object under observation.

Properties, then, are the "explicitated" result of an organising of clues, of a group of facts, which are themselves "little theories". But reality is there, doubtlessly, because it canalises our efforts to obtain knowledge in non-arbitrary directions, it causes friction, by opposing itself to our theoretical propositions, great and small, these "properties" spoken of in our languages. The transcendency of these properties, as if they were already constituted, as "ontologies", is a "flatus vocis" and surely cannot be based on Husserl's views, because it is the constitutive process of the transcendental which is at the centre of his philosophy. It is our task, when referring to different forms of scientific knowledge, to enrich and to specify this so very fuzzy word, the notion of "property" for the physical world, as well as that of mathematical property.

\subsection{Transcendence vs. transcendental constitution : Gödel vs. Husserl}

So let's move on to mathematics. In this discussion we refer to one of the most interesting among thinkers having an "ontologising" tendency (and one of the greatest mathematicians of the XXth Century), K. Gödel. Actually, Gödel also proposes a strict parallel between physical objects and mathematical concepts, although from a perspective different from ours (similarity of "ontologies" or of "independent existence"): "It seems to me that the assumption of [mathematical] objects is quite as legitimate as the assumption of physical bodies and there is quite as much reason to believe in their existence" [Gödel, 1944] ... "the properties of these concepts are something quite as objective and independent of our choice as physical properties of matter ... since we can create [them] as little as the constituent properties of matter" [Gödel, 1947]. So, physical bodies, and constituent properties of matter, as well as mathematical concepts are all preconstituted entities, possibly the ultimate building blocks, independent of or transcending the cognitive subject (not "created"). Again, even the word property, as referring to outside objective states of affairs, is used in a naïve, ordinary way, even for constituents elements, it seems, whose analysis belongs to the entangled constructions of microphysics, where the constitutive polarity "subject/object" is at the core of the modern perspective in Quantum Mechanics (indeed, since the ' $30 \mathrm{~s}$ ).

In his masterpiece about the foundations of mathematics, "The Origin of Geometry", Husserl frequently emphasises the role of the transcendental constitution of mathematical "objects". The epistemological problem they pose is, for him, a "problem of genesis", a "historical problem" (see the footnote above). Geometry, as an attempt (and mankind makes many) to make space intelligible is the result of an activity by "our communicating community"; it is "the constituted", the result of a non-arbitrary process, which grounds our constitutive hypotheses within certain regularities of the world, regularities, "donations" which impose themselves upon us; these regularities are themselves 
"already there" (the connectivity of space, isotropy, symmetries - inspiring ourselves by Riemann and Weyl.) But it is us who choose to see them.

I have a Jupiterian friend who has five legs, three eyes and a half and no, absolutely no symmetry to his body. He sees not or does not give any importance to the symmetries of light reflected by a surface, or to crystals, for example, these symmetries which are before our eyes, before his eyes; and his mathematical structures are not imbued with symmetries like ours (from Greek geometry to the dualities and adjunctions so well described in the Theory of Categories). They are rather constructed around "zurabs", an essential regularity from his perspective, but which we do not see or which we neglect. It goes likewise for colours; he sees a bandwidth beyond violet, where one can find, as a matter of fact, splendid colours. He therefore cannot appreciate this marvellous human construction, rich in history, that we call "painting" : Titian's colors are invisible for him. Just like we do not see his masterpieces, of such beautiful ultraviolet colours.

The two constructions are not arbitrary, luminous waves (or the reality we categorise as such) "are there", just as are the symmetries of crystals or of light bounces, but our active presence interacts with these elements of reality in order to choose, emphasise, correlate some of them, but not others, to gives names, not arbitrary names because they are rich in history and in meaning, to certain colour bandwidths and not to others. Moreover, our action interpolates the missing elements, proposes links by analogy, analogies derived from other experiences; it integrates a variety of acts of experience in order to create a new structure, an inexisting network between "the things" of the world. To figure out, among the regularities of the world and among the foundational acts of any form of knowledge, which ones are at the origin of mathematics is one of the tasks of the analysis of the cognitive foundations of mathematics. Husserlian phenomenal analysis may be one tool, if we do not limit ourselves to a fuzzy notion of "transcendence", but if we recover the richness of "transcendental constitution." Unfortunately, most anti-formalist mathematicians, and even the greatest of Mathematical Logic, such as Frege and Gödel, insist upon the "transcendent" ("the properties and the objects of mathematics pre-exist, just as do the properties and the objects of physics"). In fact, Gödel, while knowing Husserl, does not refer to the "genesis", to the "history" (in the sense of [Husserl, 1933]) of this constitution at the centre of our conceptual constructions ${ }^{4}$. He thus remains, in mathematics and in physics, at a stage of a realism, which neither specifies the notion of property nor that of object: it is only the objects and the properties derived "from sensations"; properties of a physics of "medium size objects" (this table, a pencil ...), physics which no longer exist, decades after work and debate in relativity, in physics of critical systems and in quantum physics. The failure of this "realist" epistemology of mathematics is parallel to the absence of an epistemology of physics.

It should be clear though that we have been mainly discussing of Gödel's "realist" position, not only as a tribute to the mathematician (of whom the work on types, in 1958, as well as that on recursion and incompleteness, in 1931, made its mark on XXth Century mathematical logic, as well as on the work of this author), but also because his philosophy is by far the most profound among philosophies of mathematical "realism/Platonism". Alain Badiou, [Badiou, 1990] emphasises the richness of this Platonism, alone, in mathematics, resembling that of ...Plato: thought envelopes the

\footnotetext{
4 See the discussions reproduced in [Wang, 1987]. [Follesdal, 1999] makes the generous effort of reading some of Husserl in Gödel. However, for Gödel, the existence of mathematical objects is as external to us as that of physical objects, in that both types pre-exist: "they are independent of our definitions and our constructions"; the intuition of mathematical objects (sets, actually) is a form of "physical perception" [Gödel, 1944, supplement in 1964], in the most naive sense of the term "perception", a sensorial "input" that reaches us as-is (we have, at many occasions, talked about the profoundness of Poincaré's sketch of a theory of perception, for example, where we find a true attempt at epistemology in mathematics, rooted in a "philosophy of nature"). There is nothing but transcendence with Gödel, even in the quotations chosen by Follesdal, without all the remainder of the phenomenal analysis characterising Husserl; transcendence without transcendental constitution, as constitutive process of knowledge, without this "Ego" that is coconstituted with the world, which is at the centre of Husserlian philosophy, particularly of its maturity.
} 
object, while the idea is "already there", but as the name of that which is thought and which would remain unthinkable if not activated within thought... Moreover, for Gödel, as we reminded, "the objective existence of the objects of mathematical intuition ... is an exact replica of the question of the objective existence of the outside world" [Gödel, 1947]. This approach, all the while bringing the question of a mathematical ontology closer to that of an ontology of physics, is far more promising than the realism common in mathematics, a funny mix of vulgar empiricism and of idealism, with the worst shortcomings of each of these two philosophies. However, the difference, relative to the approach sketched here, is given by the understanding of the object as constituted; it is not the existence of physical objects or of mathematical concepts that is at stake, but their constitution, as their objectivity is entirely in their constitutive path. It is thus necessary to take Gödel's philosophy, for what it puts into mathematical and physical relation, and to turn its head over heels, to bring it back to earth: one must not start "from above", from objects, as being already constituted (existing), but from the constitutive process of these objects and concepts. This requires a non-naive analysis of the object and of physical objectivity, as well as a non-passive theory of perception.

\subsection{Conceptual constructions: history vs. games.}

To summarise, the objects of mathematics are "outside of ourselves" (transcendent) only as much as they belong to a "constituted" which precedes our subject: they are a co-constituted, at the same time as the very intelligibility of the world, by our "living and communicating community". They are not arbitrary because they are rooted in the regularities of reality, to which are confronted our living beings in the world. They are (relative) invariants, first, of time and space, that we then develop by constructing a whole universe derived from conceptual structures, with the most stable tools of our understanding, these invariants of language and of intersubjectivity that we call "logic" and "formalisms": these as well are the result of a praxis, the practice of human reasoning, beginning with the greek agora, in human interaction. In this sense of a previous phylogenetic and historic constitution of their construction principles, and not any another, the objects of mathematics may have properties of which "we do not know", as not yet engendered properties within a more or less precisely given conceptual universe. Take the integers, for example. Once presented, by 0 and the successor operation, as the mental construct of an infinite sequence, discrete and well ordered (you can picture it, aligned from left to right in a mental space, right?), we can surely give ourselves a language (that of Peano-Dedekind, for example) and enounce an infinity of properties for the elements of this sequence which "we do not know". We will then need to exercise some "friction" between these properties, in that language, and the given construction; and to verify by the most varied methods or tools (arithmetic induction, but also complex variable functions, for example) if they are "realized" upon this well ordered, infinite structure. In other words, we need to "compare" construction principles and proof principles. It is thus like this that we may understand the essential incompleteness of the formal theory of numbers, see [Longo, 2002; Longo, 2005]. It should then be clear that this absolutely does not imply that this infinite sequence "pre-exists" as a conceptorless concept: if five stones were surely already there, at the foot of this mountain, one billion years ago, what was not there was the concept of the number of 5, something completely different, nor were the infinitary properties of that number, ordered within the infinite sequence with the others, as for example the solvability of 5th degree equations or the results of many other linguistic/algebraic constructions we know how to make; constructions that are far from being arbitrary, because rooted in a creative mix of significant conceptual methods (logico-formal, results of spatial invariants, regularities, etc.).

Also consider a variant of chess I am inventing right now: a 100x100 square, with 400 pieces that have quite varied but not arbitrary, very symmetrical finite movements, simulations of natural movements, say ... I then scatter the pieces randomly; what must be demonstrated is that the configuration thus obtained is compatible with (attainable by) the given rules. Can we say that 
configuration (a property of the game) was already there, a billion years ago? What is the meaning of that sentence? Worse, I propose a game with an infinity of squares, ordered with great originality in the three dimensions, but effective (spirals, fractals ...), I call them "spiralu numbers" or "zamburus", and give you infinitary relationships upon these conceptual objects (I describe, using words, infinite subsets, relationships upon this structure or I scatter the pieces randomly). What sense does it make to say that these properties/relationships were already there? That the compatibility of the distributions of the pieces thus obtained were already decided or were valid since ever? Surely, proof will be necessary in order to "verify" it (I prefer: to check if these distributions are "realized" upon the structure, that is to establish friction, by means of proof, between given properties in the language or the geometry of the squares and the game's construction principles). But as long as the infinitary structure, my construction, built in history, a non-arbitrary extension of a practice of squares and of order, is not posed with the rigour of its constructions principles, as the locus where to realize, by the friction of proof, this other construction given in the language of the properties to verify, what sense does it make to say that the conceptual structure and the properties of its infinite subsets 'pre-existed'? Conversely to the games which I just proposed, which are my own individual construction, the grounding in the world, within a very ancient intersubjectivity, of the concept of the number, of zero, of the successor, of the infinite well order.... gives them a "transcendent" status w.r. to my individual existence. Yet, this must not lead to forget that also these mathematical "objects" are concepts, the results of a very structured, phylogenetic and historical conceptual construction, determined by its constitutive hypotheses; they are not a "pre-existing ontology", they do not transcend our human, actually animal existence (as counting is a pre-human activity). The fact that we ignore the totality (?) of their "properties" (careful with this word) in no way demonstrates this ontology we so easily confer them: we ignore them, just as we ignore the totality of the scatterings of our whimsical chess games on the infinite chessboard above. There is no transcendence in mathematics, or, rather, there is no transcendence which is not the result of non arbitrary constitutive processes (for example, the construction of algebrico-formal enunciations or of the well-ordering of integers), constructions needing to be compared (relatively realized) with one another, by means of this "friction" between and upon conceptual structures, which is called mathematical proof. More specifically, between principles of proof (that we give ourselves, by non-arbitrary choices) and construction principles (that participate in our own cognitive determination, in the relationship to the world).

Continue, for example, and start with the construction of the integers to pass on to the rationals, as ratios of integers, modulo an equivalence of ratios; then consider the convergent sequences (of Cauchy) of these new numbers, modulo equiconvergence. There are the real numbers, constituted using a mathematical method which reconstructs and links together, in its own way, different histories, by distilling the key concepts. The real numbers do not exist, in any sense of a plausible ontology, but their constitution is as objective as are many other conceptual organisation of the world which render it intelligible to us. And they propose us a very efficient conceptual structure for the phenomenal continuum of time and space.

\section{Laws, structures and foundations.}

In his inquiry, F. Bailly, from the perspective of Physics, poses other important questions, among which I now retain those concerning the terms of "structure" and of "foundation" (see [Bailly, Longo, 2005]). What I deny is that one can identify the notion of mathematical structure with its axiomatic presentation and, then, that the analysis of proof, within these axiomatic frameworks, can be a sufficient foundational analysis. To discuss this last point, we will also speak of "Laws". Physicists sometimes confuse "formalism" with "mathematisation"; it is customary of their language. The mathematical structuration of the world, of a physical experiment, that they propose is often called "formalisation". That is quite understandable, because in what concerns the "very 
concrete" about which they are thinking (physical "reality"), the mathematical structure is surely abstract and symbolic. But with a bit of experience with the debate about the foundations of mathematics, where these terms are employed with rigour (and philosophical relentlessness, I would say), one understands that rigorous, abstract and symbolic does not mean formal (see [Longo, 2005] for a more detailed analysis of "rigorous", "abstract", and "symbolic" as differing from "formal"). In fact, a formal system must work without reference to meaning; it is constructed and manipulated thanks only to mechanical rules. These rules are also and surely used during a physico-mathematical calculus, but the formula about which the physicist thinks has nothing to do with that of logical "formalism": the formula is significative from the onset, because the physicist constructed it with permanent reference to its meaning, to his or her physical experience, he or she inserts it into a mathematical context rich with explicative connections. The physicist proposes mathematical structures to make his or her experience intelligible, the physicist does not invent a set of formal rules disconnected from the world, as would do the formalist, whose foundational analysis lies only in consistency. He or she thus proposes mathematical structures, and not formal systems. Between the two there are at least the great theorems of incompleteness, which separate structural construction principles from formal deductions.

Let's try to exemplify this distinction within mathematics themselves. Consider, as "construction principles", translations and rotations of figures constructed by rule and compass; if one fixes the unit of length, one will easily construct a segment of square root of 2 length. And there, a very first challenge for mathematical understanding: the theory of linear equations with integral coefficients, and with its formal rules of calculus, is demonstrably incomplete with regards to this construction (the segment is not a ratio between integers). With the same principles of construction, including the absence of gaps and jumps within the Euclidean continuum, construct the limit of the poligons inscribed in and circumscribed around a circle. It will then be the formal theory of rational coefficient algebraic equations, which is incomplete with regards to this construction of $\pi$. If we move on to the XXth century, it is demonstrated that the formal theory of numbers, with its proof principles, is incomplete with regards to the well order of integers as construction principle. By analogy to the role of symmetries in physics, one could say in that regard that Hilbert's conjecture of the completeness of formal Artihmetics was a mirror-symmetry hypothesis between formal language and ontologising semantics (the first accurately reflects the second). Gödel's theorem of incompleteness breaks this alleged symmetry and initiates modern logic. In more constructive and recent terms, the breaking of the symmetry between proof principles and construction principles, of an essentially geometric nature, leads us to understand the insufficiency of a sole logico-formal language as the foundation of mathematics and brings back to the centre of our forms of knowledge a constitutive mathematics of time and space, thanks to its construction principles. There is concrete incompleteness, a modern version of gödelian incompleteness, a discrepancy or breaking in provable symmetry between construction principles and proof principles (see [Longo, 2002] for a detailed analysis of proofs of some recent theorems of incompleteness).

Mathematical structures are, in fact, the result of a reconstruction which organises reality, all the while stemming from concepts, such as the pre-mathematical concept of the infinite (the theological concept, for example), or, even, from pre-conceptual practices (the invariants of memory, the experience of order, of comparison, the structurations of the visual and perceptual in general Gestalts ..., see [Longo, 2005]) which lead to a structuration, explicited in language, of these (pre-)concepts and of their relationships : the well order of the integers, the Cantorian infinite, the continuum of the real numbers, .. the notion of Riemanian manifold. The concept of infinity gets involved, because it is the result of a profound and ancient conceptual practice, as solid as many other mathematical constructions; these practices are not arbitrary and each may be understood and justified by the process of the construction of scientific objectivity to which it is related. 
After the construction of these abstract structures, that are symbolic yet rich in meaning, because they refer to the underlying practical and conceptual acts of experience, we may continue and establish axiomatic frameworks that attempt to grasp at a formal level, whose manipulation may disregard meaning. This process is important, because it adds a possible level of generality and especially highlights certain "proof principles' which enable us to work, upon these structures, by using purely logico-formal deductions, within well specified languages. But these principles are essentially incomplete, that is what the great results of incompleteness of the last 70 years, in particular the recent "concrete" ones, tell us (see [Longo, 2002]). Moreover, as we said in the introduction, the analysis of proof, particularly if this analysis is only formal, is but the last part of an epistemology of mathematics: it is also necessary to account for the constitution of the concepts and of the structures which are "manipulated" during these proofs. But there is more to this usual and fallacious identification of "axioms" with "structures", of "foundations" with "logico-formal rules". In order to understand this, let's return to physics. Husserl, in an extraordinary epistolary exchange with Weyl (see [Tonietti, 1988]), grasps a central point of relativistic physics, highlighted, particularly, by the mathematical work of Weyl (but also by the reflections of Becker, a philosopher of physics and student of Husserl, see [Mancosu\&Ryckman, 2002]). The passing from classical physics to the new relativistic framework first bases itself upon the following change in perspective: we go from causal lawfulness to the structural organisation of time and space (structural lawfulness), nay, from causal lawfulness to intelligibility as "normativity" by mathematical (geometric) structures. In fact, Riemann is at the base of this revolutionary transformation (all the while developing the ideas of Gauss). In his habilitation memoir, [Riemann, 1854], a pillar of modern mathematics and of their applications to physics, he aims to unify the different physical fields (gravitation and electromagnetism) through the geometrical structure of space. He throws out the hypothesis that the local structure of space (its metric, its curvature) may be "linked to the cohesive forces between bodies". "Divination" Weyl will call it in 1921, for it is effectively the viewpoint peculiar to this geometrisation of physics which at least begins with Riemann, finds its physical meaning with Einstein, and, with Weyl, its modern mathematical analysis.

It thus seems to me that the attempt to mathematise the foundational analysis of mathematics by only referring to the "laws of thought" is comparable to a reconstruction of the unique, absolute classical universe in physics, with its Newtonian laws. It is not a priori laws that regulate mathematics, but they do constitute themselves as structures, conceptual plays, that are not arbitrary. The "cohesive forces", in mathematics, would correspond to an "interactive dynamic of meaning", a structuration of concepts and of deduction itself.

In category theory, for example, we propose a new conceptual structure, by novel objects (invariants) and morphisms (transformations); we link it to other structures by using functors, that we analyse in terms of transformations ("natural", their technical name), all the while following/reconstructing the open dynamic of mathematics, of which the unity manifests itself through these reciprocal translations of theories (interpretation functors). And the relative (functorial) interpretation s relate the ongoing conceptual constructions (categories): unity is an ongoing conquest and not given by a pre-existing set-theoretic background universe. Moreover, certain of these categories have strong properties of closure, a bit like rational numbers that are closed for multiplication and division, as real numbers are for particular limits ... One of the logically interesting properties, among many others, is "small completeness", that is, the closure with regards to products which interpret the universal quantification, among which, in particular, second-order quantification (quantification upon collection of collections). Through this device, some categories confer mathematical meaning to the challenges of impredicativity [Asperti, Longo, 1991], the great bogeyman of "stratified" worldviews and of logic (formal certitudes constructed upon elementary and simple building blocks, one level independent of the other ...). The world however seems to build itself upon essential circularities, from the merest dynamical system (three 
bodies interacting in a gravitational field) or the local/global interaction (non-locality) in quantum physics, up to the "impredicative" unity of any living organism, of which the parts have no meaning and are out of place outside of the organism as a whole (see [Longo, 2000], [Bailly, Longo, 2003]). Maybe the emergence of that which is new, in physics, in biology, only takes place under the presence of strong circularities, sorts of internal interactions with complex systems.

Mathematics are thus not a logico-formal deduction, nicely stratified from these axioms of set theory that are as absolute as Newton's universe, but are structurations of the world, abstract and symbolic, doubtless, yet not formal, because significant; their meaning is constructed in a permanent resonance to the very world they help us understand. They then propose collections of "objects" as conceptual invariants, of which the important thing is the individuation of the transformations which preserve them, exactly like (iso-)morphisms and functors preserve categorical structures (properties of objects of a category).

There are no absolutes given by logical rules, beyond the world and the cognitive subject, by definite rules (but then why not those of scholastics or of Euclid's key rule: "a part always has less elements than does the whole", which is false in the case of our infinite sets?), but there is a dynamic of structures (of categories), emergent from a mathematical practice, then linked by those interpretation functors which unify them, which explain the ones by the others, which confer them meaning within a "reflexive equilibrium" of theories (and of categories, particularly those which correspond to deductive systems [Lambek, Scott, 1986], [Asperti, Longo, 1991]).

Surely there is a temporality in the construction of the meaning we confer to the world through mathematics; and it is a "rich" temporality, because it is not that of sequential deduction, of Turing Machines: it is closer to the evolution of space distributed dynamical type systems (see [Bailly, Longo, 2004]). We must let go of this myth of pre-existing "laws of thought" and immerse mathematics into the world while appreciating its constitutive dynamics of which the analysis is an integral part of the foundational project. The laws or "rules" of mathematical deduction, which are surely at the centre of proof, are themselves also the constituted of a praxis, of language, as invariants of the reasoning and of the practice of proof itself.

The foundation, so, as the constitutive process of a piece of knowledge, constructed responsively to the world, the physical world and that of our sensations. But ... where does this process begin? It is surely not a case of reascending "to the mere stuff of perception, as many positivists assert", since physical objects are "intentional objects of acts of consciousness " [Weyl, 1918b]. There is a very Husserlian remark, a constitution of objects which we have called a conceptual "découpage" (cutting-off). And this découpage is performed (and produced) by the mathematical concept, conscious mental act towards the world. Then, reasoning, sometimes rooted in a whole different practice, in the language of social interaction, that of the rules of logical coherence or of the aesthetic of symmetries, for example, generates new mathematical concepts, which may themselves, but not necessarily, propose new physical objects (positrons, for example, derived from electrons by a pure symmetry between equations in microphysics).

The autonomy of mathematics, thanks to the generativity of reasoning, even of the formal type (calculus for example), is indubitable, there lies their predictive force in physics. The integration of these different conceptual dimensions, of these different praxis (geometrical structuration of the world, logical and formal deduction, even far removed from any physical meaning), also confers mathematics their explicative and normative character with regards to reality: one goes from a physical invariant, from space, let's say, to another by purely logico/formal means (an algebraic transformation applied to this invariant and which preserves it, a symmetry ...) and a new physical object is thus proposed. The physical proof will be a new experience to invent, with instruments to be invented.

Obviously, in this grounding of our sciences into the world, perception also plays an essential role, but we must then develop a solid theory of perception, rooted in a cognitive science that 
allows to go far beyond the positivist's "passive perception", of which Weyl speaks about. We shall return to this point.

The approach we propose, of course, causes the loss of the absolute certitude of logico-formal, decidable proof. But we know, since Gödel, that any formal theory, be it slightly ambitious and of which the notion of proof is decidable, is essentially incomplete. So logicism's and formalism's "unshakeable certainties" (the absolute certification of proof) are lost ...since a long time. There remains the risk of the construction of scientific objectivity, thoroughly human, even in mathematics, the adventure of thought which constitutes its own structures of the intelligibility of the world, by the interaction with the former and with the thoughts of others. The risk, for example, of acknowledging the foundational role of the well ordering of integers, by a geometric judgement constituted in history, action, language and intersubjectivity, in order to certify the coherence of Artithmetics [Longo, 2002; 2005].

\section{Subject and objectivity.}

In various works, Weyl develops a very interesting philosophical analysis concerning the passage in physics from the subjective to the objective, on the basis of references to his own mathematical works in relativity theory. This analysis is emphasised by [Mancosu, Ryckman, 2002], who refer mostly to [Weyl, 1918b ; 1927]. The importance of Weyl's remarks obviously extends way beyond the philosophical stakes in physics and in mathematics, because it touches upon a central aspect of any philosophy of knowledge, the tension between the "cult of the absolute" and "relativism". Husserl seeks to move beyond this split in all of his work and in his reading of the history of philosophy (see, for example, [Husserl, 1956]). XXth Century physics can provide tools for contributing to that debate, and those are Weyl's motivations.

For Weyl, immediate experience is "subjective and absolute", or, better, it claims to be absolute; the objective world, conversely, that the natural sciences "crystallise out of our practical lives ... this objective world is necessary relative". So, it is the immediate subjective experience which proposes absolutes, while the scientific effort towards objectivity is relativising, because "it is only presentable in a determined manner (through numbers or other symbols) after a coordinate system is arbitrarily introduced in the world. This oppositional pair : subjective-absolute and objective-relative seems to me to contain one of the most fundamental epistemological insights that can be extracted form natural sciences".

Following his works in relativity, Weyl thus gives a central role to reference frames. The subject lays, chooses, a reference frame and in this manner organises time and space. That choice is the very first step performed by the knowing subject. But the operation of measurement, by means of its own definition, also implies the subject: any physical size is relative to (and set by) a "cognising ego". The passage to objectivity is given, in quantum physics, by the analysis of "gauge invariants", for example, one of Weyl's great mathematical contributions to this field: they are given as invariants in relation to the passing from one detection and measurement system to another. More generally, the passage from subjectivity to scientific objectivity implies the explicit and explicitated choice of a reference frame, including for mathematical measurements and invariants.

Weyl thus emphasises, in Husserlian fashion, that any object in the physical world is the result of an intentional act, of the awareness "of a pure, sense giving ego". For both thinkers, it is a matter of the Cartesian "Ego", to which Husserl so often returns to, which "is, since it thinks"; and it is, because, as a consciousness, it has "objects of consciousness" (consciousness is "intentional", it has an "aim"). It is the subject, this conscious Cartesian "Ego", that chooses the reference frame and who, afterwards, is set aside. It poses the origin, the 0 and the measurement, and it mathematically structures time and space (as Cantor-Dedekind continuum, for example, or as a Riemannian manifold with its curvature tensors); by that act (the construction of a space as 
mathematical manifold), it poses a framework of objectivity, independently of the subject, objectivity nevertheless consciously relativised to that choice. Because the choice of "viewpoint", of the frame, is relativising and breaks the absolute characteristic of the subject before the passage to scientific objectivity; this passing of subjectivity, which claims to be absolute, to relativising objectivity, is the meaning of the scientific approach central to relativity. Just as it is very well put in [Mancosu, Ryckman, 2002], "The significance of [Weyl's] "problem of relativity" is that objectivity in physics, that is, the purely symbolic world of the tensor field of relativistic physics, is constituted or constructed via subjectivity, neither postulated nor inferred as mind-independent or transcendent to consciousness". But this symbolic world of mathematics is in turn itself the result of an interaction of the knowing subject(s), within intersubjectivity, with the regularities of the world, these regularities which we see and which are the object of intentional acts, of a view directed with "fullness and willing", as Husserl and Weyl say ${ }^{5}$.

The subject is thus at the origin of scientific knowledge, and it is with the subject that any mathematical construction begins. However, it will be necessary to push the analysis of the subject's role further: today we can pose the problem of objectivity at the very centre of the knowing subject, because this subject is not the psychological subject, which is also disputed by the seekers of the absolute, of transcendental truths, of configurations or properties which are already there, true prior to any construction/specification, even in my infinite chessboard or in the sequence of integers. In fact, it is a question of the "cognitive subject", of this «Ego» that we share as living, biological creatures, living in a common history that is co-constituted with the world, at the same time as its activity in the world. There is the next issue we will have to deal with, in the dialog with cognitive sciences, basing ourselves on non-naive (and non passive) theories of perception, on theories of the objective co-constitution of the subject. The scientific analysis of the subject must, by these means, underline what is common to subjective, psychological variability: more than a simple "intersection of subjectivities" it is a question of grasping in that way what lies behind individual variabilities, what directs them and allow them to communicate and to understand/construct the world together.

\footnotetext{
${ }^{5}$ The profoundness of Weyl's philosophy of sciences is extraordinary and his philosophy of mathematics is but a part of it (a small one). I found quite misleading, with regards to this profoundness and its originality, the many attempts of many, including some leading "predicativists" to make him into a predecessor of their formalist philosophy of mathematics. Shortly, in [Weyl, 1918] (a remarkable Husserlian analysis of the phenomenal continuum of time and space) Weyl also feels concerned with the problem of "good definitions", a problem that preoccupied all mathematicians of the time (including Poincaré and Hilbert, of course): the XIXth Century was a great period for mathematics, but, so very often,.... what a confusion, what a lack of rigour! Particularly, it was necessary to watch out for definitions that may have implied circularities, such as impredicative definitions. Weyl noticed that Russell's attempt to give mathematics a framework of "stratified" certitudes does not work ("he performs a hara-kiri with the axiom of reductiveness", [Weyl, 1918]). In the manner of the great mathematician he was, Weyl proposed, in a few pages, a formally "predicativist" approach that works a thousand times better than that of Russell with his theory of types. An approach (an interesting exercise in clarification) that Weyl will never follow in his mathematical practice; to the contrary, in [Weyl, 1918], he criticises, quite a few times, Hilbertian formalism of which the myth of complete formalisation "trivialises mathematics" and comes to conjecture the incompleteness of formal arithmetics (!). Feferman, in [Feferman, 1987], took up these ideas only slightly brushed upon by Weyl (and not the predicatively incoherent heaviness of Russell's theory of types), to make it into an elegant and coherent predicative formal theory for Analysis. Remarkable technical work, but accompanied by an abusive and quite incomplete reading of Weyl's philosophy, which is a much broader Philosophy of Natural Sciences, never reduced to stratified predicativisms nor their corresponding formal or logicist perspectives (I expressed these critical opinions, preceded by a punctual analysis of the very interesting technical aspects, in a report, [Journ. Symb. Logic, 53/3, 1993], see also [Longo, 1989 ; 1999]) ; the biased reading of history is common with formalism, beginning with the presentation of Euclid as the first of formalists ... unable however of demonstrating in a formally correct manner - in short, as Hilbert would - the first theorem of the first book [Heath, 1908], see next note.
} 
Foundational analysis, in mathematics and in physics, must therefore propose a scientific analysis of the cognitive subject and, then, highlight the objectivity of the construction of knowledge within its referenctial systems or reference frames.

In what concerns the foundations of mathematics, a process analogous to this "choice of reference frame" is well explicitated, in Category Theory, by choosing the right "topos" (as referential category for a logic or with an "internal logic" [Johnstone, 1977]), to relate, through interpretation functors, other categorical constructions, in a dynamic of these structures by which we give mathematical meaning to the world (algebraic, geometrical, manifolds' categories ...). This has nothing to do, as we have already emphasised, with the absoluteness of the axioms of set theory, a Newtonian universe that has dominated mathematical logic and that has contributed for a century to the separation of mathematical foundations from epistemology and from the philosophy of natural sciences. That was a matter, indeed, of an absolute, that of sets, intuition of which is compared, by the "realists" of mathematical philosophy, to the perception of physical objects (quite naively described in its passivity), sets and objects also being transcendent, with their properties all "pre-existing, since unknown" [Gödel, supplement to 1947]. A typical example of that which Husserl, de Ideen, and Weyl (taken up by Becker, see [Mancosu, Ryckman, 2002]) call the "dogmatism" of those who speak of absolute reality, infinite list of already constituted properties, constituted before any pre-conscious and conscious access, before the shared practices in our communicating community.

\section{From intuitionism to a renewed constructivism.}

Quite fortunately, within the same mathematical logic, we begin to hear different voices: "Realism: No doubt that there is reality, whatever this means. But realism is more than the recognition of reality, it is a simple-minded explanation of the world, seen as made out of solid bricks. Realists believe in determinism, absoluteness of time, refuse quantum mechanics: a realist cannot imagine "the secret darkness of milk". In logic, realists think that syntax refers to some pre-existing semantics. Indeed, there is only one thing which definitely cannot be real: reality itself" [Girard, 2001]. The influence of Brouwer, leader of intuitionism, and of Kreisel, as well as the mathematical experience with intuitionnist systems, is surely present in the mathematical work and in the rare philosophical reflections of Girard, but without the slip, characteristic of Brouwer, into a senseless solipsism, nor with the a priori limitations of our proof tools. Moreover, time and space are included in Girard's proof analysis: the connectivity, the symmetries of proof as network, time as irreversible change in polarity in [Girard, 1987; 2001], have nothing to do with "time as secreted by clocks" (his expression), the time of sequential proof, of Turing machines, which is beyond the world (see [Bailly, Longo, 2004] and [Longo, 2002b]).

Brouwer's intuitionism, among the different trends in the philosophy of mathematics (formalist, platonician realist, intuitionnist), is possibly the only foundational analysis that has attempted to propose an epistemology of mathematics (and a role for the knowing subject). The discreet sequence of numbers, as trace of the passing of time in memory ([Brouwer, 1948], see also [Longo, 2003]), is posed as constitutive element of mathematics. It is exactly this vision of mathematics as conceptual construction that has made Weyl appreciate Brouwer's approach for a long time. In fact, the analyses of the mathematical continuum for Brouwer and Weyl (as well as for Husserl, see [Weyl, 1918], [Tonietti, 1988], [Longo, 1999]) are quite similar in many respects. However, Weyl had to distance himself from Brouwer, during the $20 \mathrm{~s}$, when he realised that the latter excessively limits the tools of proof in mathematics and does not know how to go beyond the "psychological subject", to the point of renouncing the constitutive role of language and of intersubjectivity and to propose a "languageless mathematics" (a central theme of Brouwer's solipsism, see [Brouwer, 1948], [vanDalen, 1991]). 
Conversely, and as we have tried to see, the relativity problem for Weyl, as passage from "causal lawfulness" to "structural lawfulness" in physics, as well as play between subjectivityabsolute and objectivity-relative, is at the centre of an approach that poses the problem of knowledge in its unity, particularly as relationship between physical objectivity and the mathematical structures that make time and space intelligible, thanks, among other things, to language. All the while following Weyl, we have made a first step towards an extension of foundational analysis in mathematics by a cognitive analysis of what should precede purely logical analyses: only the last segment is without doubt constituted by the logico-formal analysis of proof. But upstream there remains the problem of the constitution of structures and of concepts, a problem which is strictly related to the structuration of the physical world and to its objectivity. The project of a cognitive analysis of the foundations of mathematics thus requires an explicitation of the cognitive subject. As a living brain/body unit, dwelling in intersubjectivity and in history, this subject outlines the objects and the structures, the spaces and the concepts common to mathematics and to physics, on the phenomenal veil. In short, parallel constitutive history, in physics, begins with perception as action: we construct an object by an active viewing, by the presence of all of our body and of our brain, as integrator of the plurality of sensations (Merleau-Ponty's "vision as palpation by sight', perception as the result of a comparison between sensorial input and a hypothesis performed by the brain, [Berthoz, 1997]). In fact, any invariant is an invariant in relation to one or more transformations, so in relation to action. And we isolate, we "single out", invariants from the praxis that language, the exchange with others, forces us to transform into concepts, independently, as communicables, from the constitutive subject, from invariants constituted with others, with those who differ from us but who share the same world with us, and the same type of body. From the act of counting, the appreciation of the dimentionless trajectory - dimentionless since it is a pure direction -, we arrive at the mathematical concepts of number, of unidimensional line and, then, of point, [Longo, 2005]. Invariants quite analogous to the physical concepts of energy, force, gravitation, electron ... The latter are the result of a similar process, they are conceptual invariants which result from a very rich and "objective" praxis, that of physics, inconceivable without a close interaction with mathematics. They organise the cues that we select through perception and through action upon the world, through our measurement instruments; the geometrical structuration of those invariants is the key organising tool, because it explicitates in time and space our action and our comprehension.

Individual and collective memory is an essential component to this process constitutive of the conceptual invariants (spatial, logical, temporal ...). The capacity to forget in particular, which is central to human (and animal) memory, helps us erase the "useless" details; useless with regards to intentionality, to a conscious or unconscious aim. The capacity to forget thus contributes in that way to the constitution of that which is stable, of that which matters to our goals, which we share: in short, to the determination of these invariant structures and concepts, which are invariant because filtered of all which may be outside our intentional acts of knowledge, [Longo, 1999]. Their intercultural universality is the result of a shared or "sharable" praxis, in the sense that these invariants, these concepts, may very well be proposed in one specific culture (think about Greek geometry or Arabic algebra), but their rooting in fundamental human cognitive processes (our relationship to measurement and to the space of the senses, basic counting and ordering ...) make them accessible to other cultures. This widening of a historic basis of usage is not neutral, it may require the blotting out of other experiences specific to the culture which assimilates them, but confers them this universality that accompanies and which results from the maximal stability and conceptual invariance specific to mathematics. But this universal is posed with relation to human experience and does not mean absolute; it is itself a cultural invariant, between cultures that take shape through interaction. Because universality is the result of these communicating communities and because historical demise is also a factor; oblivion or expulsion from mathematics of magical numbers, of zombalo structures ... of that which does not have the generality of method and results 
we call, a posteriori, mathematical. As for the mathematical organisation of space, both physical and sensible, it begins very early, probably as soon as space is described by gesticulation and words, or with the spatial perspective and width of the pictorial images of Lascaux, 20000 years ago, or from the onstart of the play of Euclid's rigid bodies which structures geometrical space. Euclid's axiomatics indeed summarise the minimal actions, indispensable to geometry, with their rule and compass, their construction and measurement instruments: "trace a straight line from one point to another", " extend a finite line to a continuous line", "construct a circle from a point and a distance" ... (note that all these constructions are based and/or preserve symmetries). His first theorem is the "vision of a construction" (in Greek, theorem means "sight", it is like a "theatre"): he instructs how to "construct an equilateral triangle from a segment", by symmetric tracing with a compass ${ }^{6}$.

This history leads to Weyl's symmetries, regularities of the world which impose themselves (donations that, in this sense, pre-exist or that reality imposes us), but that we see or decide to see. We then transform them into concepts and choose to pose them as organising criteria of reality, even in microphysics, far removed from sensorial space. But now we turn to [Bally, Longo, 2005a] for more work in this direction.

\section{REFERENCES}

(Preliminary or revised versions of Longo's papers and book are downloadable from: http://www.di.ens.fr/users/longo or Search : Google : Giuseppe Longo).

Asperti A., Longo G. Categories, Types and Structures, M.I.T. Press, 1991.

Badiou A. Le Nombre et les nombres, Seuil, 1990.

Bailly F. "About the emergence of invariances in physics : from "substantial" conservation to formal invariance", in Quantum Mechanics, Mathematics, Cognition and Action, (M. Mugur-Schächter and A. van der Merwe, Eds.), Kluwer 2002.

Bailly F., Longo G. "Objective and Epistemic Complexity in Biology" Invited lecture, Proceedings of the International Conference on Theoretical Neurobiology, (N. D. Singh, ed.), National Brain Research Centre, New Delhi, INDIA, pp. 62 - 79, 2003.

Bailly F., Longo G. "Space, Time and Cognition: From The Standpoint of Mathematics and Natural Sciences" in Mind and Causality, (Peruzzi ed.), Benjamins, Amsterdam, pp. 149-199, 2004.

Bailly F., Longo G. "Incomplétude et incertitude en mathématiques et en physique", actes du colloque Il pensiero filosofico di Giulio Preti, (Parrini, Scarantino eds.), Guerrini ed associati, Milano, pp. 305 340, 2004a.

Bailly F., Longo G. "Sui rapporti fra i fondamenti della Matematica e della Fisica: dialogo" di prossima pubblicazione, (Castellana ed.), Lecce, 2005.

Bailly F., Longo G. "Causalites et symetries dans les sciences de la nature. Le continu et le discret mathematiques" paraître, (Joinet, ed.) Presses de la Sorbonne, Paris, 2005a.

Bell J. A Primer in Infinitesimal Analysis, Cambridge U.P., 1998

Berthoz A. Le sens du mouvement, Od. Jacob, 1997

Bitbol M. L'aveuglante proximité du réel, Flammarion 2000

Brouwer L. "Consciousness, Philosophy and Mathematics", 1948, in Collected Works vol. 1 (Heyting ed.), North Holland, 1975.

van Dalen D. "Brouwer's dogma of languageless mathematics and its role in his writings" Significs, Mathematics and Semiotics (Heijerman ed.), Amsterdam, 1991.

\footnotetext{
${ }^{6}$ That's what the first theorem of the first book is and the point constructed during proof is the result of the intersection of two lines traced using a compass. Its existence has not been forgotten, as claimed by the formalist reading of this theorem, it is constructed: Euclid's geometry presupposes and englobes a theory of the continuum, without gaps or jumps (see Parmenides and Aristotle).
} 
Feferman, S. "Weyl Vindicated: "Das Kontinuum" 70 Years Later", Proceedings of the Cesena Conference in Logic and Philosophy of Science, 1987

Follesdal D. "Gödel and Husserl", in : Naturalizing Phenomenology (J. Petitot, F. Varela, B. Pachoud, JM. Roy, Eds.) Stanford University Press 1999.

van Fraassen B. Lois et symétrie, Vrin 1994.

Frege G. The Foundations of Arithmetic, 1884 (english transl. Evanston, 1980)

Girard J.-Y. "Linear Logic" Theoretical Comp. Sci., 50 (1-102), 1987

Girard J.-Y. "Locus Solum", Special issue, Mathematical Structures in Computer Science, Cambridge U.P., vol.11, n.3, 2001

Gödel K. "Russell's mathematical logic" in The philosophy of B. Russell (Schlipp ed.), 1944 (reprinted in Philosophy of mathematics; selected readings (Benacerraf, Putnam, eds), Prentice-Hall, 1964).

Gödel K. "What is Cantor's Continuum Problem?", Amer. Math. Monthly, 54, 1947 (reprinted in Philosophy of mathematics; selected readings (Benacerraf, Putnam, eds), Prentice-Hall, 1964).

Heath T.L. The Thirteen Books of Euclid's Elements, Cambridge Univ. Press, 1908

Husserl E. L'origine de la Géométrie, 1933 (trad. fran. PUF, 1962)

Husserl E. Erste Philosophie, M. Nijhoff, 1956 (trad. it. G. Piana, Guerini, Milano, 1989)

Johnstone P. Topos Theory. Academic Press, 1977.

Lambek J., Scott P.J. Introduction to higher order categorical logic, Cambridge University Press 1986

Lobachevskij N. Nouveaux principes de la Géométrie, 1856.

Longo G.. "Some aspects of impredicativity: Weyl's philosophy of mathematics and today's Type Theory" in Logic Colloquium 87 (European Summer Meeting of the A.S.L.), pp. 241--274. Invited Lecture (Ebbinghaus et al., eds). North-Holland, 1989.

Longo G. "The Mathematical Continuum, from Intuition to Logic" in : Naturalizing Phenomenology (J. Petitot, F. Varela, B. Pachoud, J-M. Roy, Eds.) Stanford University Press 1999.

Longo G. "Cercles vicieux, Mathématiques et formalisations logiques". Conférence Invitée, parue dans Mathématiques, Informatique et Sciences Humaines, n. 152, 2000.

Longo G. "On the proofs of some formally unprovable propositions and Prototype Proofs in Type Theory" Invited Lecture, Types for Proofs and Programs, Durham, (GB), Dec. 2000; Lecture Notes in Computer Science, vol 2277 (Callaghan et al. eds), pp. 160 - 180, Springer, 2002.

Longo G. "The Constructed Objectivity of Mathematics and the Cognitive Subject", in Quantum Mechanics, Mathematics, Cognition and Action (M. Mugur-Schachter ed.), Kluwer, 2002a.

Longo G. "Laplace, Turing and the "imitation game" impossible geometry: randomness, determinism and programs in Turing's test". Invited lecture, Conference on Cognition, Meaning and Complexity, Univ. Roma II, June 2002b. (version française, Intellectica, n. 35/2, 2002b).

Longo G. "Mémoire et objectivité en mathématiques" in : Le réel en mathématiques, Colloque de Cerisy, 1999 (P. Cartier et N. Charrauds Eds.), Editions rue d'Ulm, 2003.

Longo G. "The Cognitive Foundations of Mathematics: human gestures in proofs and mathematical incompleteness of formalisms" (Okada ed.), Keio Univ. Press, 2005.

Manacosu P., T. Ryckman, "Mathematics and Phenomenology. The correspondence between Oskar Becker and Hermann Weyl", Philosophia Mathematica vol. 10, pp. 130-202, 2002.

Parrini P. Conoscenza e Realta', Laterza, 1995.

Riemann B. On the hypothesis which lie at the basis of Geometry, 1854 (english transl. by W. Clifford, Nature, 1873.

Salanskis J-M. L'herméneutique formelle, Ed. CNRS 1991.

Tonietti T. L. "Four letters of E. Husserl to H. Weyl and their context" in Exact Sciences and their philosophical foundations, Peter Lang, Frankfurt, 1988

Wang H. Reflections on Kurt Gödel, M.I.T. Press, 1987

Varela F. Autonomie et connaissance, Seuil 1989

Verlet L. La malle de Newton, Gallimard 1993

Weyl H. Das Kontinuum, 1918

Weyl H. Raum, Zeit, Materie, 1918b

Weyl H. Philosophy of Mathematics and of Natural Sciences, 1927 (english transl., Princeton University Press, 1949). 